\section{AB011. Surgical management of myasthenia gravis}

\author{
Eric Dominic Roessner ${ }^{1}$, Alexander $\operatorname{Marx}^{2}$
}

${ }^{1}$ Division of Surgical Oncology and Thoracic Surgery, Department of Surgery, ${ }^{2}$ Institute of Pathology, University Medical Centre Mannheim, Heidelberg University, Heidelberg, Germany

Correspondence to: Prof. Dr. Eric Dominic Roessner, FEBTS. Division of Surgical Oncology and Thoracic Surgery, Department of Surgery, University Medical Centre Mannheim, Heidelberg University, Heidelberg, Germany. Email: eric.roessner@umm.de.

Abstract: Since Ferdinand Sauerbruch performed a subtotal thyroidectomy with concomitant subtotal thymectomy with a subsequent clinical improvement of myasthenia gravis (MG) in 1912, surgeons try to improve the approaches, extend and strategy of thymectomies. The effector mechanism of MG are autoantibodies which target the neuro-muscular junction. In the 1970s the detection of acetylcholine receptor antibodies supported the theory of an autoimmune disease. In the 2000s the muscle specific kinase was identified as an additional autoantibody. Just recently low-density lipo-protein receptor related protein 4 (LRP4) was postulated to be a potential autoantibody target. The interaction of these antibodies leads to numerous effects on the transmission of the electric signal from the nerve to the muscle cells with a complex interaction of different cells of the immune response originating from the thymus gland. These interactions are evident in non thymomatous MG as well as MG with thymoma. In latter, additional paraneoplastic mechanism are discussed. Interestingly, the incidence of $\mathrm{MG}$ is highest in $\mathrm{AB}$ and $\mathrm{B} 2$ thymoma patients, of whom about one quarter suffer from paraneoplastic MG. Whereas the indication for thymectomy is clear in cases of thymoma plus MG, the indication for thymectomy for non-thymomatous MG remains in parts controversial. Up to 2016 the evidence base for this indication was as low as level III. In 2016 Wolfe et al. published a first of its kind land mark RCT on thymectomy in MG. Although methodology was controversial in some areas it was the first RCT to show the positive effects of this procedure in MG. The primary outcomes were the time-weighted average quantitative Myasthenia Gravis score over a 3-year period and the time-weighted average required dose of prednisone over a 3-year period. In a follow up publication, the study group could show a continuous benefit in a 5 -year follow up. The findings of these RCTs support the conclusion of many other non-randomized trial or retrospective analysis. Nevertheless, many aspects of modern thymic surgery proclaimed remain unclear and need further high-level evidence. This includes the surgical approach and the extent of resection, as well as the definition of the patient cohort benefiting the most from the procedure. Although the Wolfe study investigates only open transsternal approaches, with a patient recruitment period of 2006 to 2012, many surgeons believe that the modern minimal invasive approaches are at least equal in the treatment effect with fewer complications. These minimal invasive approaches include extended transcervical approaches, video-assisted thoracoscopic surgery (VATS) approaches either in a classic fashion transintercostal or subxiphoid as well as robotic approaches. All these approaches proved feasible and safe in expert hands but failed to show superiority in a board use. The extend of the resection in the study was defined as en bloc resection of all mediastinal tissue that could anatomically contain gross or microscopic thymus (or both). In many publications this kind of a resection is called an extended thymectomy although the terms thymectomy and extended thymectomy are used inconsistently throughout the literature. Many surgeons believe that the removal of all mediastinal tissue is indicated, since many studies showed ectopic thymic tissue outside the distinct gland and spread in mediastinal fat. But there is no level II or higher evidence supporting this. A higher incidence of phrenic nerve injuries with consecutive diaphragmatic paralysis might be associated with this extended resection, resulting in serve pulmonary complication in MG patients, as well as other rare but major complications like vessel injuries. Regarding the patient cohort suitable for surgical treatment the Wolfe study included patients with a disease onset of less than 3 years, an age of 18 to 60 years, a serum acetylcholinereceptor-antibody level of more than $1.00 \mathrm{nmol}$ per liter (elevated levels of 0.50 to $0.99 \mathrm{nmol}$ per liter were accepted if the diagnosis was confirmed by a positive edrophonium test, abnormal repetitive nerve stimulation, or abnormal single-fiber electromyography), and a Myasthenia Gravis Foundation of America clinical classification of II to IV. Participants could be taking appropriate anticholinesterase therapy with or without oral glucocorticoids. Exclusion criteria were thymoma on computed tomography or magnetic resonance imaging of the chest. All these criteria go inline with the guidelines for this disease prior to the 
study and have not changed since then. Again, only weak evidence supports the criteria. We conclude on the basis of available evidence that surgery for $M G$ is beneficial for sero-positive patient between 18-60 years with a short duration of the disease. In the daily practice of the authors the robotic extended thymectomy is used routinely. In addition, we offer this operation to AChR-sero-negative patients after an in-depth informed consent process.
Keywords: Thymectomy; video-assisted thoracoscopic surgery (VATS); robotic-assisted thoracoscopic surgery (RATS); transsternal

doi: 10.21037/shc.2019.AB011

Cite this abstract as: Roessner ED, Marx A. Surgical management of myasthenia gravis. Shanghai Chest 2019;3:AB011. 